Article

\title{
Molecular Dynamics Simulations for Resolving Scaling Laws of Polyethylene Melts
}

\author{
Kazuaki Z. Takahashi ${ }^{1,2, *}$, Ryuto Nishimura ${ }^{2}$, Kenji Yasuoka ${ }^{2}$ and Yuichi Masubuchi ${ }^{3}$ \\ 1 Multi-Scale Soft-Matter Simulation Team, Research Center for Computational Design of Advanced \\ Functional Materials, National Institute of Advanced Industrial Science and Technology (AIST), Central 2, \\ 1-1-1 Umezono, Tsukuba, Ibaraki 305-8568, Japan \\ 2 Department of Mechanical Engineering, Keio University, 3-14-1 Hiyoshi, Kohoku-ku, Yokohama, \\ Kanagawa 223-8522, Japan; r.nishimura2016@gmail.com (R.N.); yasuoka@mech.keio.ac.jp (K.Y.) \\ 3 National Composite Center, Nagoya University, Furocho, Chikusa, Nagoya 464-8630, Japan; \\ mas@nuap.nagoya-u.ac.jp \\ * Correspondence: kazu.takahashi@aist.go.jp; Tel.: +81-29-861-2972 \\ Academic Editor: Patrick Underhill \\ Received: 13 November 2016; Accepted: 4 January 2017; Published: 12 January 2017
}

\begin{abstract}
Long-timescale molecular dynamics simulations were performed to estimate the actual physical nature of a united-atom model of polyethylene (PE). Several scaling laws for representative polymer properties are compared to theoretical predictions. Internal structure results indicate a clear departure from theoretical predictions that assume ideal chain statics. Chain motion deviates from predictions that assume ideal motion of short chains. With regard to linear viscoelasticity, the presence or absence of entanglements strongly affects the duration of the theoretical behavior. Overall, the results indicate that Gaussian statics and dynamics are not necessarily established for real atomistic models of PE. Moreover, the actual physical nature should be carefully considered when using atomistic models for applications that expect typical polymer behaviors.
\end{abstract}

Keywords: molecular dynamics simulations; polymer melts; scaling law

\section{Introduction}

Rheological predictions for specific polymer materials must be improved for advances in polymer-based technologies. Fundamentally, this problem originates from the complexity of polymer structure, dynamics, and physical properties. For example, processes that govern polymer properties change drastically over different time scales. Importantly, phenomena that occur over a wide range of timescales are closely related to each other; i.e., structure and dynamics at the micro-scale can affect properties at the meso- and macro-scales [1-3]. Many polymer models have been developed for each scale and have been studied for many years [4-12]. At the micro- and meso-scales, molecular dynamics (MD), Monte Carlo, and metadynamics simulations that utilize molecular models of polymers are promising approaches [13-16]. In particular, MD simulations can estimate entangled polymer dynamics via explicit equation-of-motion calculations of intra- and intermolecular interactions. Recent advances in computer power have enabled a wide range of MD simulations for polymers [17-25]. It is now possible that the actual physical nature of each molecular model can be precisely evaluated and discussed. The universality of polymer dynamics predicted by theoretical approaches that use single chains and mean-fields has not been established for actual molecular models. For example, Gaussian statistics assumed in Rouse models is not observed for molecular models unless the molecular weight is sufficiently high. Non-Gaussian statistics affects the dynamics, which then deviate from predictions of the Rouse model. While these deviations are often concealed in scaling laws, this issue should be carefully considered. 
An all-atomistic (AA) molecular model is potentially the most precise classical model; however, the equilibrating of AA polymer systems is much more difficult than that of united-atom (UA) polymer systems. For instance, Harmandaris and Kremer reported that the dynamics of AA polystyrene (PS) systems was about 120 times slower than that of UA PS systems [26]. It implies that the precise estimation of actual physical nature for AA polymer systems is challenging, even though using the recent computational power. Therefore, UA models are widely used to perform MD simulations of polymers. For UA models of the common polymer polyethylene (PE), the anisotropic united atom (AUA) [27], optimized potentials for liquid simulations (OPLS)-UA [28] and transferable potentials for phase equilibria force field (TraPPE)-UA [29] models are widely accepted [30-36]. MD simulations using UA PE models have been recently performed for a wide range of polymer nanocomposites [37,38], polymer interfaces [39-41], ring polymers [42], the nucleation of polymer droplets [43], the Fermi-Pasta-Ulam problem in realistic systems [44,45], and a better understanding of macroscopic mechanical properties [21,46-49]. However, polymer properties at long timescales need to be carefully evaluated for validation of models. Here, we performed long-time MD simulations using the TraPPE-UA PE model. To examine the actual physical nature of the model, several scaling laws for representative polymer properties were estimated and compared to some theoretical predictions.

\section{Methodology}

MD simulations of PE melts were performed using the TraPPE-UA PE model [29]. Two different types of united atoms $\left(\mathrm{CH}_{3}\right.$ and $\left.\mathrm{CH}_{2}\right)$ were defined in a $\mathrm{PE}$ chain, whose non-bonded interactions were described by Lennard-Jones 12-6 potentials. All bond lengths were kept rigid using the LINCS algorithm [50], whereas a harmonic potential was used to describe bond angle bending. Standard torsional potentials were used to describe rotations along bonds in the aliphatic backbone. These dihedral potentials counted also for the 1-4 non-bonded interactions. Using this UA model, we performed atomistic MD simulations for PE melts with molecular weight, $M$, ranging from 422.8 to $2807 \mathrm{~g} / \mathrm{mol}$. The molecular dynamics package GROMACS [51] was used for effective computing. The different PE systems that have been simulated are presented in Table 1. Initial well-equilibrated atomistic structures were obtained by long-time MD simulations (over $100 \mathrm{~ns}$ ) with intermittent pressure rising and temperature falling processes at constant particle number, pressure, and temperature ensembles. The equilibration of systems was confirmed from comparison with previous reports [36]. The long-time MD simulations for product runs were performed with a constant particle number, volume, and temperature ensemble using the Nosé-Hoover thermostat $[52,53]$. To attain the precise relaxation dynamics quickly, the density $\rho$ and temperature $T$ were set to $0.650 \mathrm{~g} / \mathrm{cm}^{3}$ and $500 \mathrm{~K}$, respectively. Non-bonded interactions were cut off beyond $1.2 \mathrm{~nm}$. The Verlet leapfrog integrator [54] was used with three-dimensional periodic boundary conditions and a time step of $2 \mathrm{fs}$. For $M=422.8-983.9 \mathrm{~g} / \mathrm{mol}$, a total of $5 \times 10^{7}$ time steps (=100 ns) of equilibrium simulations were performed for three independent initial structures. For $M=1405-2106 \mathrm{~g} / \mathrm{mol}$, a total of $2.5 \times 10^{8}$ time steps $(=500 \mathrm{~ns})$ in equilibrium simulations were performed for six independent initial structures. For $M=2807 \mathrm{~g} / \mathrm{mol}$, a total of $4 \times 10^{8}$ time steps $(=800 \mathrm{~ns})$ in equilibrium simulations were performed for six independent initial structures.

Table 1. United-atom polyethylene systems studied in the present work ( $\rho=0.65 \mathrm{~g} / \mathrm{mol}$ and $T=500 \mathrm{~K})$.

\begin{tabular}{cccc}
\hline $\boldsymbol{M}$ (g/mol) & No. of chains & Simulation time (ns) & No. of initial structures \\
\hline 422.8 & 1000 & 100 & 3 \\
703.4 & 600 & 100 & 3 \\
983.9 & 428 & 100 & 3 \\
1405 & 300 & 500 & 6 \\
2106 & 200 & 500 & 6 \\
2807 & 150 & 800 & 6 \\
\hline
\end{tabular}




\section{Results and Discussion}

\subsection{Static Properties}

In Flory's theory [55] of polymer melts, equilibrium chains with uniform lengths are expected to satisfy ideal Gaussian statics. However, the chain length required to satisfy the theory is non-trivial and depends on the polymer architecture and model description. To evaluate Gaussian statics in polymer melts, the scaling law relationship between the number of beads per chain $N(\propto M)$. In this work, $N$ is equal to the number of carbons in the PE chain), and the mean-square end-to-end distance $\left\langle R^{2}\right\rangle$, and the mean-square radius of gyration $\left\langle R_{\mathrm{G}}^{2}\right\rangle$ were computed. The terms $\left\langle R^{2}\right\rangle$ and $\left\langle R_{\mathrm{G}}^{2}\right\rangle$ are given by:

$$
\begin{aligned}
\left\langle R^{2}\right\rangle & =\left\langle\boldsymbol{R}^{2}\right\rangle \equiv\left\langle\left(\boldsymbol{r}_{N}-\boldsymbol{r}_{1}\right)^{2}\right\rangle, \\
\left\langle R_{\mathrm{G}}^{2}\right\rangle & =\frac{1}{N}\left\langle\sum_{j=1}^{N}\left(\boldsymbol{r}_{j}-\boldsymbol{r}_{\mathrm{c} . \mathrm{m} .}\right)^{2}\right\rangle, \\
\boldsymbol{r}_{\mathrm{c} . \mathrm{m} .} & =\frac{1}{N} \sum_{j=1}^{N} \boldsymbol{r}_{j},
\end{aligned}
$$

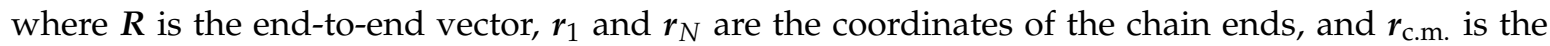
center-of-mass coordinate of the chain. In Flory's theory, $\left\langle R^{2}\right\rangle$ and $\left\langle R_{\mathrm{G}}^{2}\right\rangle$ scale as $M$. Figure 1 shows the results for (a) $\left\langle R^{2}\right\rangle-M$, (b) $\left\langle R_{\mathrm{G}}^{2}\right\rangle-M$ scalings, and (c) the ratio $\left\langle R^{2}\right\rangle /\left\langle R_{\mathrm{G}}^{2}\right\rangle$ with respect to $M$. In general, universal polymer behavior is observed for $N>N_{\mathrm{e}}$, where $N_{\mathrm{e}}$ is the critical length that indicates onset of chain entanglement [1,2]. In the UA PE model, $N_{\mathrm{e}} \sim 80$ was estimated from the primitive pass analysis [56-58]. Thus, curve fitting was done for the $\left\langle R^{2}\right\rangle-M$ and $\left\langle R_{\mathrm{G}}^{2}\right\rangle-M$ at $M>M_{\mathrm{e}}$, where $M_{\mathrm{e}}$ corresponds to $N_{\mathrm{e}} \cdot\left\langle R^{2}\right\rangle$ and $\left\langle R_{\mathrm{G}}^{2}\right\rangle$ scale with $M^{1.052}$ and $M^{1.121}$, respectively. These differ by $5.2 \%$ and $12 \%$, respectively, from values expected for ideal chains. The discrepancy was observed for short-chain conditions, indicating non-Gaussian statics. The ratio $\left\langle R^{2}\right\rangle /\left\langle R_{\mathrm{G}}^{2}\right\rangle$ deviates from the behavior of an ideal chain. The slow convergence to the ideal value $\left(\left\langle R^{2}\right\rangle /\left\langle R_{\mathrm{G}}^{2}\right\rangle=6\right)$ is observed with increasing $M$. This can be problematic when PE chains are expected to satisfy the typical polymer behavior (i.e., static universality).

The static structure factor $S(q)$ of an individual chain reveals the internal structure of polymer melts, and is given by:

$$
S(q)=1+\frac{1}{N}\left\langle\sum_{j \neq k} \exp \left[-i q \cdot\left(\boldsymbol{r}_{j}-\boldsymbol{r}_{k}\right)\right]\right\rangle
$$

where $q$ is a spatial frequency equal to $2 \pi / r$, and $r$ is an intra- or intermolecular distance. The fractal scattering of $S(q) \sim q^{-1 / v}$ is expected to be equal to $q^{-2}(v=1 / 2)$ and be independent of chain length. Figure 2 shows the results for $S(q)$. The unique $S(q)$ shape is observed for $q>2.0 \mathrm{rad} / \mathrm{nm}$; however, the fractal scattering is clearly different from that expected for an ideal chain. This reveals that the expected cancellation of dispersion forces for polymer melts [55] is not entirely satisfied in actual molecular models. The fractal scattering of $S(q)$ at $2.0 \mathrm{rad} / \mathrm{nm}<q<10 \mathrm{rad} / \mathrm{nm}$ was estimated to be $q^{-1.342}(v=0.7452)$, which differs by $33 \%$ from the expected value. These results indicate that non-Gaussian statics dominate the internal structure of polymer melts, irrespective of chain length.

The radial distribution function $g(r)$ reveals the local structure of polymer melts, and is given by:

$$
g(r)=\frac{1}{4 \pi r^{2} \Delta r \rho} \frac{\left\langle\sum_{j} n_{j}(r)\right\rangle}{N-1},
$$

where $n_{j}(r)$ is the number of beads in the region between $r$ and $r+\Delta r$ in the molecule $j$. The term $n_{j}(r)$ can be defined for the total, intermolecular, and intramolecular contributions. Figure 3 shows 
the results for the intramolecular contribution of $g(r)$, which describes the probability for beads in the same chain to meet each other. The $g(r)$ for total and intermolecular contributions exhibit only small differences with respect to $M$ (data not shown).

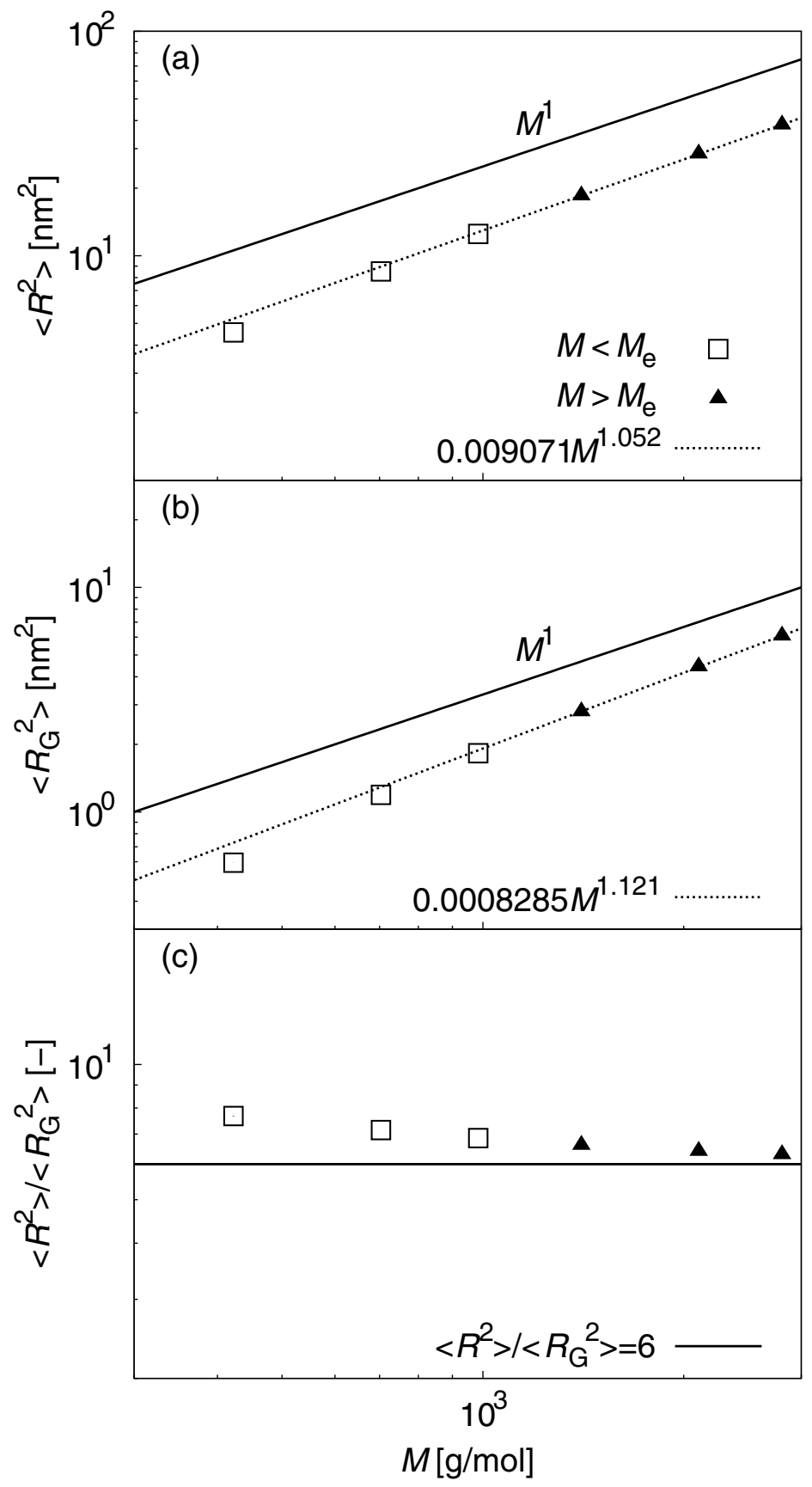

Figure 1. Results for (a) $\left\langle R^{2}\right\rangle-M$; (b) $\left\langle R_{\mathrm{G}}^{2}\right\rangle-M$ scalings; and (c) the ratio $\left\langle R^{2}\right\rangle /\left\langle R_{\mathrm{G}}^{2}\right\rangle$ with respect to $M$. Fitting curves for data at $M>M_{\mathrm{e}}$ are also plotted. $\left\langle R^{2}\right\rangle$ and $\left\langle R_{\mathrm{G}}^{2}\right\rangle$ scale with $M^{1.052}$ and $M^{1.121}$, respectively. These differ by $5.2 \%$ and $12 \%$, respectively, from values expected for ideal chains. The ratio $\left\langle R^{2}\right\rangle /\left\langle R_{\mathrm{G}}^{2}\right\rangle$ deviates from ideal chain behavior. The slow convergence to the ideal value $\left(\left\langle R^{2}\right\rangle /\left\langle R_{\mathrm{G}}^{2}\right\rangle=6\right)$ is observed with increasing $M$. 


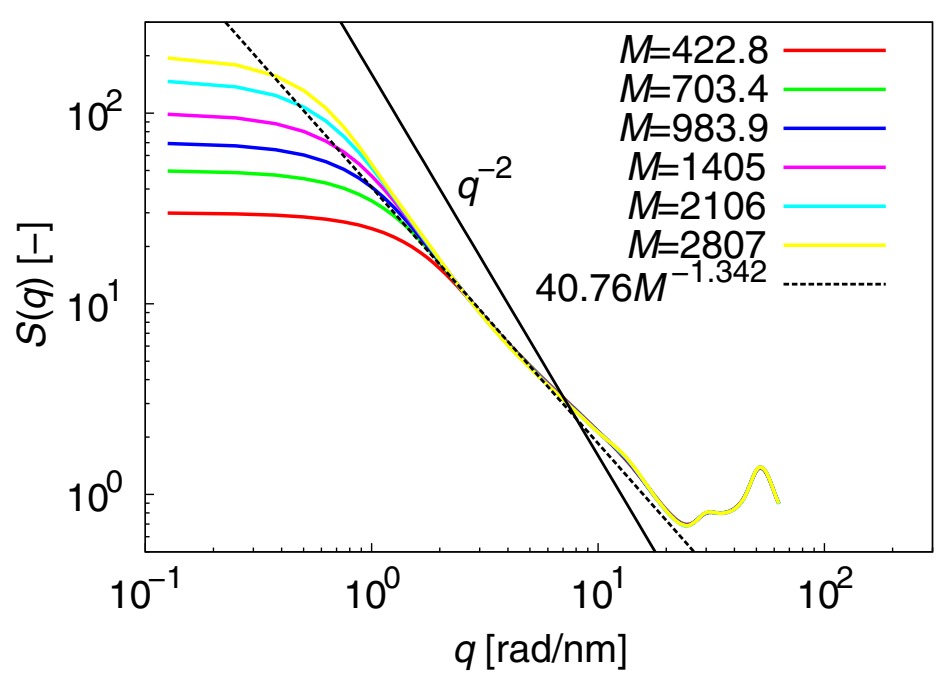

Figure 2. Results for $S(q)$. The unique $S(q)$ shape is observed for $q>2.0 \mathrm{rad} / \mathrm{nm}$; however, the fractal scattering is clearly different from that expected for an ideal chain. The fractal scattering of $S(q)$ at $2.0 \mathrm{rad} / \mathrm{nm}<q<10 \mathrm{rad} / \mathrm{nm}$ was estimated to be $q^{-1.342}(v=0.7452)$, which differs by $33 \%$ from the expected value.

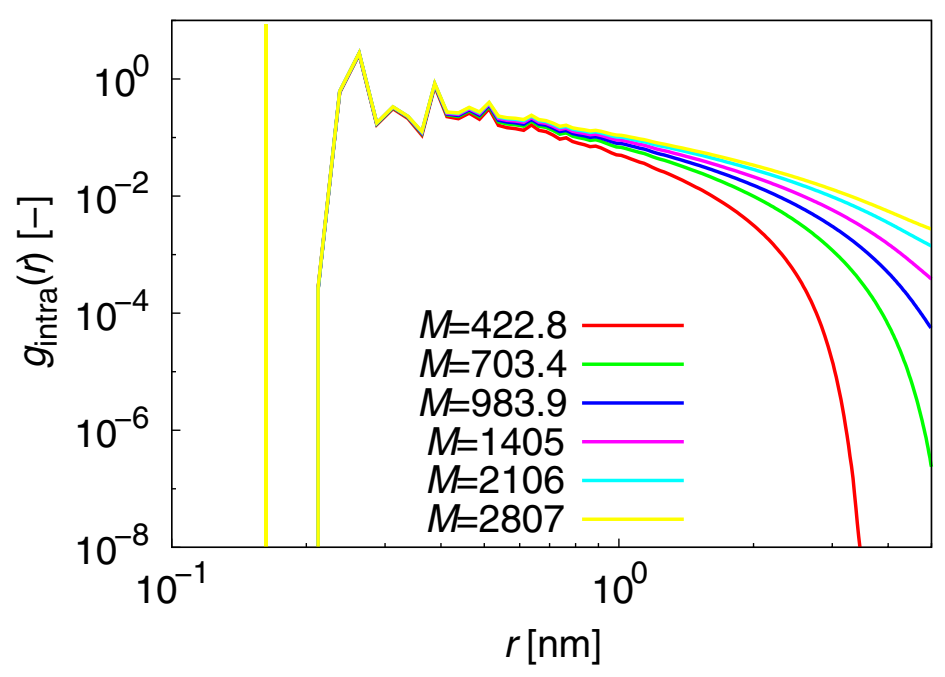

Figure 3. Results for the intramolecular contribution of $g(r)$.

\subsection{Dynamic Properties}

From the Rouse [59] and reptation models [1], the scaling law relations between $N$ and the end-to-end relaxation time $\tau_{\mathrm{R}}$, and the diffusion coefficient $D$, are approximately given by:

$$
\begin{aligned}
& \tau_{\mathrm{R}} \propto\left\{\begin{array}{ll}
N^{2} & \left(N<N_{\mathrm{e}}\right) \\
N^{3} & \left(N>N_{\mathrm{e}}\right)
\end{array},\right. \\
& D \propto \begin{cases}N^{-1} & \left(N<N_{\mathrm{e}}\right) \\
N^{-2} & \left(N>N_{\mathrm{e}}\right)\end{cases}
\end{aligned}
$$


Evaluating whether the atomistic PE model follows Equations (6) and (7) is important for molecular modeling of polymer melts.

The term $\tau_{\mathrm{R}}$ can be estimated from the time-correlation function of the end-to-end vector $C(t)$ :

$$
C(t)=\frac{\langle\boldsymbol{R}(t) \cdot \boldsymbol{R}(0)\rangle}{\left\langle R^{2}\right\rangle} .
$$

$C(t) \sim \exp \left(-t / \tau_{\mathrm{R}}\right)$ is expected, independent of the chain length. Figure 4 is a semi-logarithmic plot of $C(t)$. For the range $0.1<C(t)<1 / e, C(t)$ has linear slopes, irrespective of chain length. This indicates that $C(t)$ clearly satisfies the above expected relation and that $\tau_{\mathrm{R}}$ can be accurately estimated at $0.1<C(t)<1 / e$.

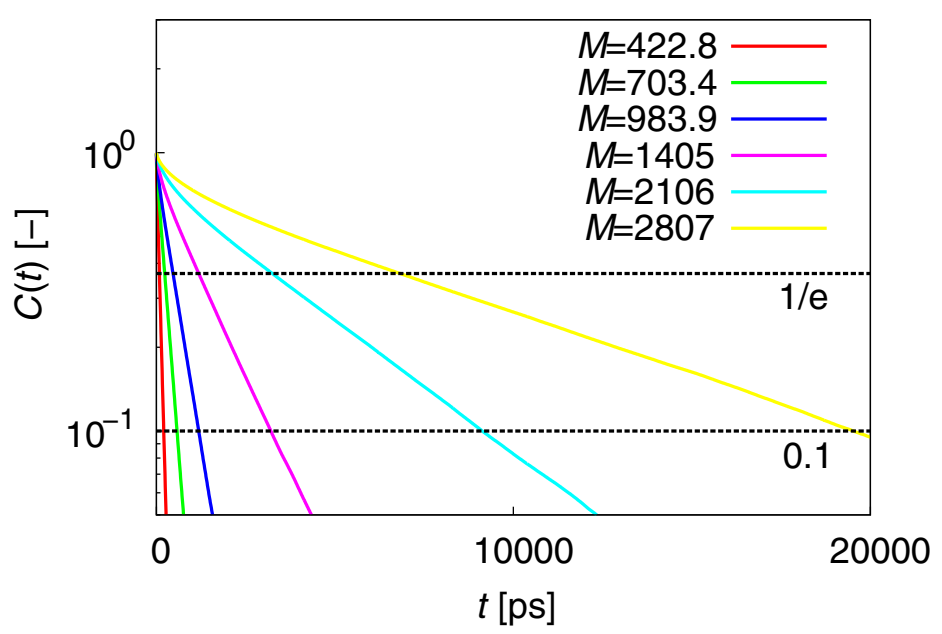

Figure 4. Semi-log plot of $C(t)$. For the range $0.1<C(t)<1 / e, C(t)$ have linear slopes, irrespective of chain length.

The term $\tau_{\mathrm{R}}$ was estimated from $C(t)$. Figure 5 shows the results for $\tau_{\mathrm{R}}-M$ scaling. For $M<M_{\mathrm{e}}$, $\tau_{\mathrm{R}}$ scales with $M^{2.1}$, which is $5 \%$ different from the scaling exponent predicted from the Rouse theory. This indicates that the motion of the PE chain at $M<M_{\mathrm{e}}$ is close to ideal chain motion. For $M<M_{\mathrm{e}}$, $\tau_{\mathrm{R}}$ scales with $M^{2.7}$, which is $10 \%$ different from the scaling exponent predicted from the reptation theory. This indicates that the motion of the PE chain at $M>M_{\mathrm{e}}$ is also close to ideal chain motion. However, it should be noted that a $\tau_{\mathrm{R}} \propto M^{3.4}$ scaling relation is expected from experimental data [2].

The term $D$ can be estimated from the mean-square displacement (MSD) of the chain center $g_{1}(t)$ :

$$
g_{1}(t)=\left\langle\left[\boldsymbol{r}_{\text {c.c. }}(t)-\boldsymbol{r}_{\text {c.c. }}(0)\right]^{2}\right\rangle,
$$

where $r_{\text {c.c. }}$ is the coordinate of the chain center. From the Rouse and reptation models, the scaling-law sequence for the MSD is roughly expected to be:

$$
g_{1}(t) \sim\left\{\begin{array}{ll}
t^{1} & \left(t<\tau_{0}\right) \\
t^{1 / 2} & \left(\tau_{0}<t<\tau_{\mathrm{e}} \sim N_{\mathrm{e}}^{2}\right) \\
t^{1 / 4} & \left(\tau_{\mathrm{e}}<t<\tau_{N} \sim N^{2}\right) \\
t^{1 / 2} & \left(\tau_{N}<t<\tau_{\mathrm{R}} \sim N^{3} / N_{\mathrm{e}}\right) \\
t^{1} & \left(t>\tau_{\mathrm{R}}\right)
\end{array},\right.
$$


where $\tau_{0}$ is a specific short time, and $\tau_{\mathrm{e}}$ and $\tau_{N}$ are the Rouse relaxation times that correspond to $N_{\mathrm{e}}$ and $N$, respectively. Figure 6 plots $g_{1}(t)$. The expected shape of $g_{1}(t)$ from Equation (10) for $M=2807 \mathrm{~g} / \mathrm{mol}$ is also plotted. The results of $g_{1}(t)$ at $M=2807 \mathrm{~g} / \mathrm{mol}$ have approximately the same scaling-law, as expected. However, the threshold values for Equation (10) are unclear from the MSD results.

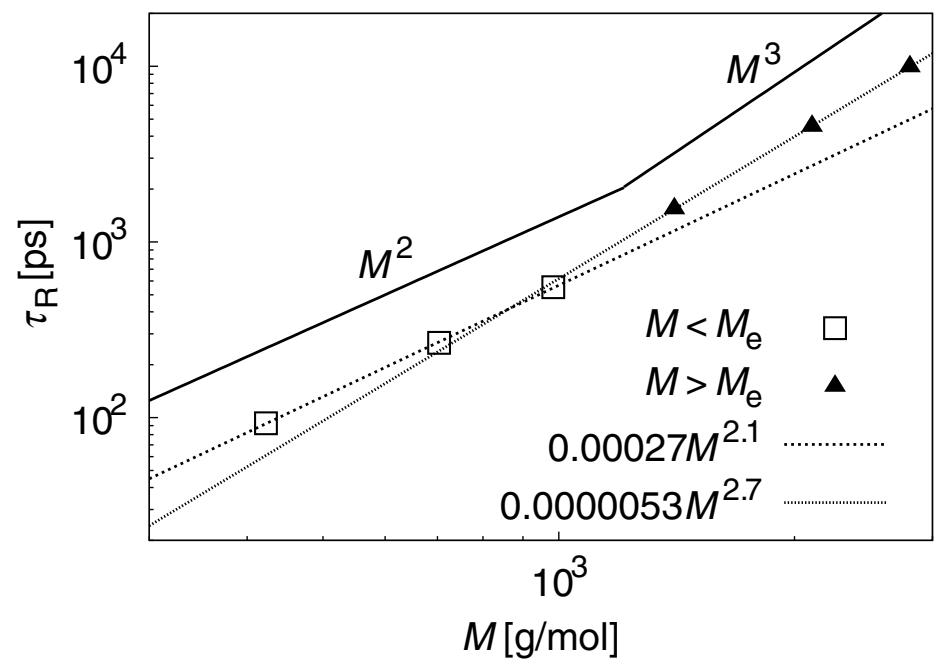

Figure 5. $\tau_{\mathrm{R}}-M$ scaling law. For $M<M_{\mathrm{e}}, \tau_{\mathrm{R}}$ scales with $M^{2.1}$, and is $5 \%$ different from the scaling exponent predicted from the Rouse theory. For $M<M_{\mathrm{e}}, \tau_{\mathrm{R}}$ scales with $M^{2.7}$ and is $10 \%$ different from the scaling exponent predicted from the reptation theory.

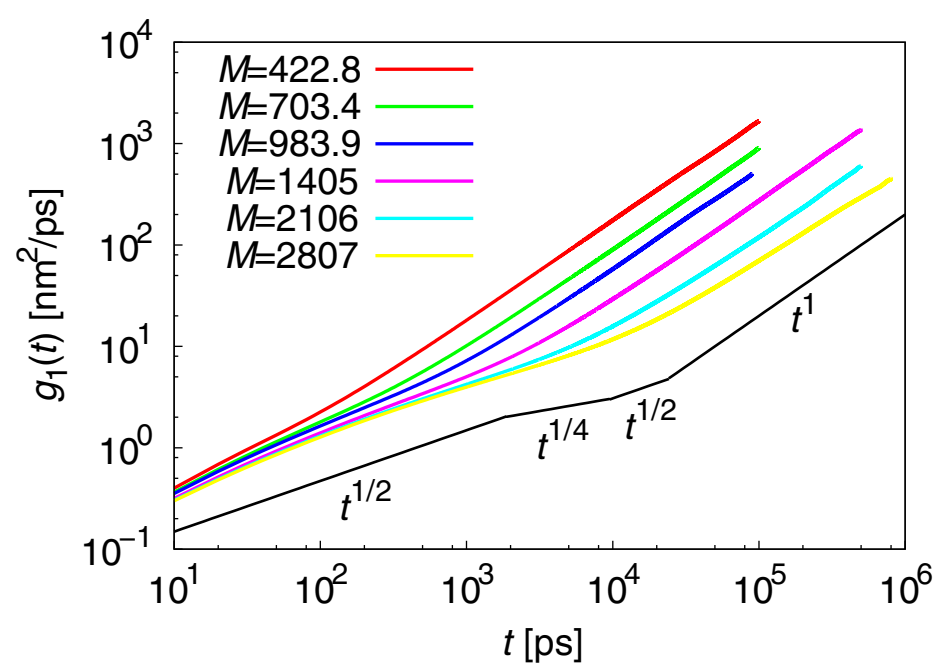

Figure 6. Results for $g_{1}(t)$. The expected shape of $g_{1}(t)$ from Equation (10) for $M=2807 \mathrm{~g} / \mathrm{mol}$ is also plotted. The results of $g_{1}(t)$ at $M=2807 \mathrm{~g} / \mathrm{mol}$ roughly have the same scaling-law, as expected. However, the threshold values for Equation (10) are unclear from the mean-square displacement results. 
The term $D$ was estimated from $g_{1}(t)$. Figure 7 plots the results for $D-M$ scaling. For $M<M_{\mathrm{e}}$, $D$ scales with $M^{-1.4}$, while the expected value is $\sim M^{-1}$. This large discrepancy indicates that the motion of short chains does not reflect Gaussian dynamics and contradicts the results of $\tau_{\mathrm{R}}$ at $M<M_{\mathrm{e}}$ shown above. For the atomistic PE model, the relation between $\tau_{\mathrm{R}}$ and $D$ established from the Rouse theory is not satisfied. For $M<M_{\mathrm{e}}, \tau_{\mathrm{R}}$ scales with $M^{-2.1}$, and is $5 \%$ different from the scaling exponent predicted from the reptation theory. This indicates that PE chain motion at $M>M_{\mathrm{e}}$ is close to ideal chain motion.

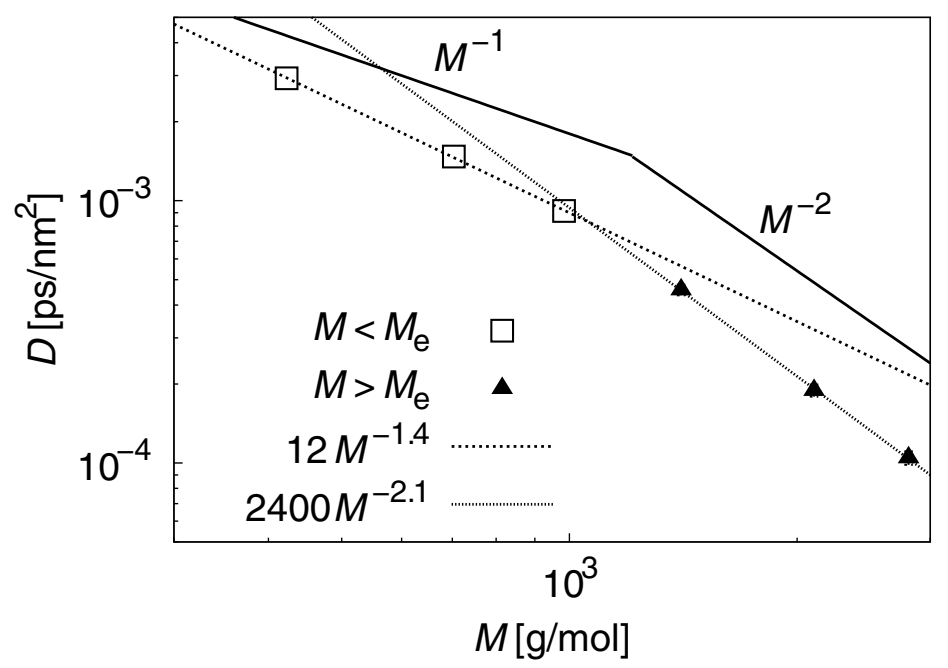

Figure 7. Results for the $D-M$ scaling law. For $M<M_{\mathrm{e}}, \tau_{\mathrm{R}}$ scales with $M^{-1.4}$, while the expected value is $\sim M^{-1}$. For $M<M_{\mathrm{e}}, \tau_{\mathrm{R}}$ scales with $M^{-2.1}$, which is $5 \%$ different from the scaling exponent predicted from the reptation theory.

The relaxation moduli $G(t)$ reveal the viscoelastic behavior of polymer melts and are given by:

$$
G(t)=\frac{V}{k_{\mathrm{B}} T}\left\langle\sigma_{\alpha \beta}(t) \sigma_{\alpha \beta}(t)\right\rangle,
$$

where $\sigma_{\alpha \beta}$ are the off-diagonal stress components $x y, x z$, and $y z$. Figure 8a shows $G(t)(\log -\log$ plot). Figure 8b plots $G(t) t^{1 / 2}$ (semi-log plot) to illustrate deviations from the $G(t)$ of Rouse theory that scale as $\sim t^{-1 / 2}$. The semi-log plot has two advantages: (i) The $y$-axis can be compressed so that all deviations can be shown in less than one decade; and (ii) all the deviations from the Rouse theory are easily seen as deviations from the horizontal line. For $M=2106$ and $2807 \mathrm{~g} / \mathrm{mol}$, the peaks indicate entanglement at long times. The deviation from the Rouse theory that indicates onset of entanglement was observed at $10 \mathrm{ps}$. Therefore, the Rouse behavior can only be seen in the short-time range (5-10 ps). For $M=1405 \mathrm{~g} / \mathrm{mol}$, the tendency is similar; however, the entanglement is weak (i.e., the peak is small). For $M=703.4$ and $983.9 \mathrm{~g} / \mathrm{mol}$, the Rouse behavior is observed at $2-100 \mathrm{ps}$. These results indicate that the duration of the Rouse behavior highly depends on the presence or absence of entanglement. Long-time MD simulation is a powerful way to obtain detailed results for an atomistic PE model. In contrast, the bead-spring model cannot reveal short duration Rouse behavior of entangled PE chains [60]. This illustrates the limitations of simplified models and the effectiveness of atomistic MD simulations. 


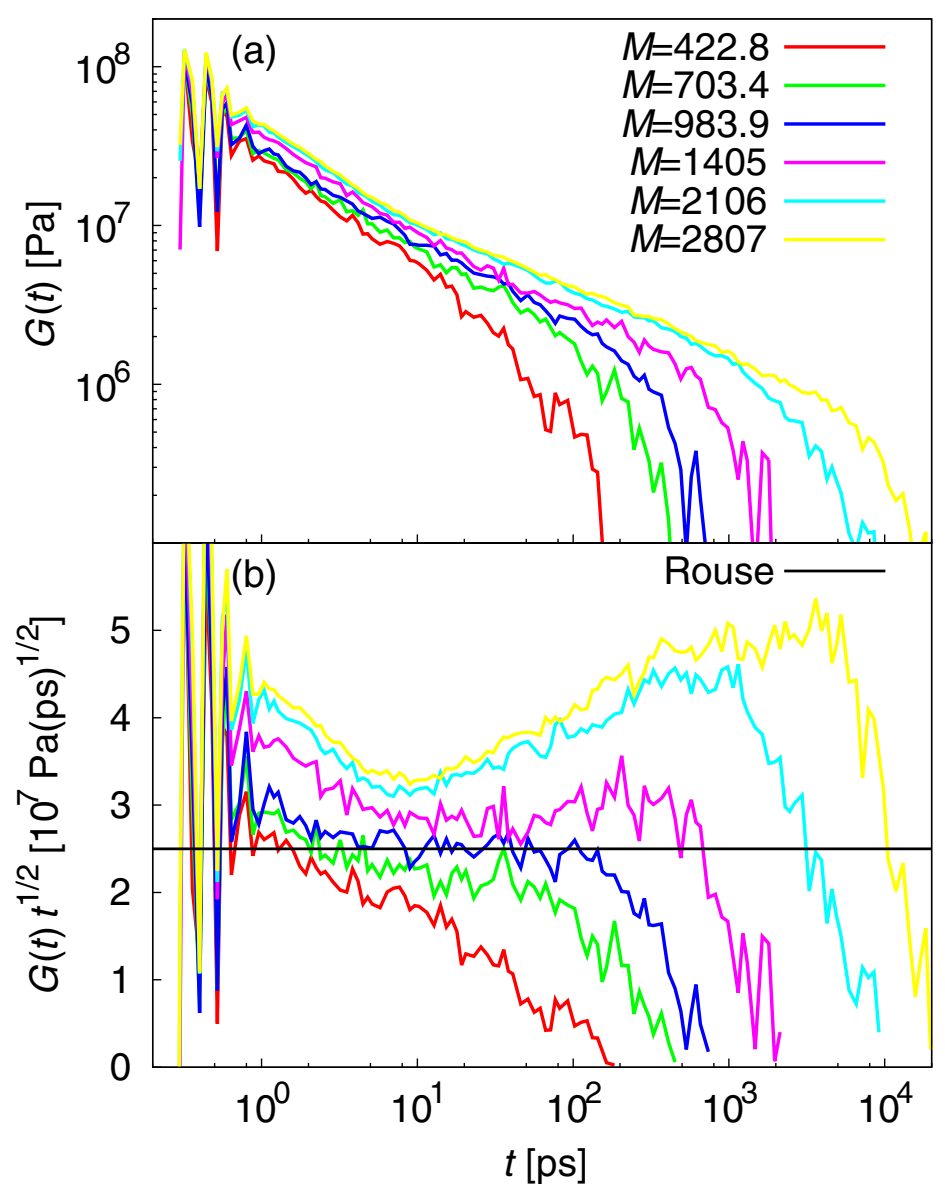

Figure 8. (a) $\log -\log$ plot of $G(t)$; and (b) semi-log plot of $G(t) t^{1 / 2}$. For $M=2106$ and $2807 \mathrm{~g} / \mathrm{mol}$, peaks that indicate entanglement were observed at long times. Deviation from the Rouse theory that indicates onset of entanglement was observed at $10 \mathrm{ps}$. The Rouse behavior can only be seen over the short-time range (5-10 ps). For $M=1405 \mathrm{~g} / \mathrm{mol}$, the tendency is roughly the same; however, the entanglement is weak (i.e., the peak is small). For $M=703.4$ and $983.9 \mathrm{~g} / \mathrm{mol}$, Rouse behavior is observed at 2-100 ps.

\section{Conclusions}

We performed long-time MD simulations using an atomistic model of PE. To examine the actual physical nature of the model, representative polymer properties were estimated. Scaling laws were compared to theoretical predictions. For the internal structure, results for $\left\langle R^{2}\right\rangle$ and $\left\langle R_{\mathrm{G}}^{2}\right\rangle$ indicate that the atomistic PE model for short chains does not satisfy Gaussian statics. The results for $S(q)$ show a clear deviation from theoretical predictions that assume ideal chain statics, irrespective of chain length. With regard to chain motion, $\tau_{\mathrm{R}}$ satisfies the prediction from the Rouse theory, while $D$ clearly deviates from it. Thus, the relationship between $\tau_{\mathrm{R}}$ and $D$ in the Rouse theory is not satisfied. Regarding linear viscoelasticity, the presence or absence of entanglement strongly affects the duration of Rouse behavior. Entangled PE chains have a very short duration. These actual physical attributes should be carefully considered when using the atomistic model for applications that expect typical polymer behavior.

In general, the theoretical predictions do not reflect strictly the microscopic factors for estimating properties. For instance, the Rouse model and Flory's theory of polymer melts rely on the single-chain motion and Gaussian statistics; however, the actual physical nature of polymers strongly affects from the many-body effect which usually cannot be expressed using the simple Gaussian statistics. This microscopic effect becomes small as the time and length scales increase but is never ignorable. MD simulations can deal with the many-body effect explicitly. This can be thought of as the main reason of the discrepancy between MD simulations and theoretical predictions. 
Overall, different atomistic models may lead to different results [26,36]. The AA PE model has the potential to improve the results. The slow dynamics of AA models is a bottleneck; however, the massively parallel computing may resolve this problem.

Acknowledgments: Kazuaki Z. Takahashi was partially supported by the Japan Society for the Promotion of Science (JSPS) Grants-in-Aid for Scientific Research (KAKENHI) Grant Number 16H06071.

Author Contributions: Kazuaki Z. Takahashi, Kenji Yasuoka, and Yuichi Masubuchi designed the study; Kazuaki Z. Takahashi and Ryuto Nishimura performed the study; and Kazuaki Z. Takahashi wrote the paper.

Conflicts of Interest: The authors declare no conflict of interest.

\section{References}

1. Doi, M.; Edwards, S.F. The Theory of Polymer Dynamics; Oxford University Press: Oxford, UK, 1988; Volume 73.

2. Ferry, J.D. Viscoelastic Properties of Polymers; John Wiley \& Sons: Hoboken, NJ, USA, 1980.

3. Masubuchi, Y. Simulating the flow of entangled polymers. Annu. Rev. Chem. Biomol. Eng. 2014, 5, 11-33.

4. Baaden, M.; Marrink, S.J. Coarse-grain modelling of protein-protein interactions. Curr. Opin. Struct. Biol. 2013, 23, 878-886.

5. Brini, E.; Algaer, E.A.; Ganguly, P.; Li, C.; Rodríguez-Ropero, F.; van der Vegt, N.F. Systematic coarse-graining methods for soft matter simulations-A review. Soft Matter 2013, 9, 2108-2119.

6. Everaers, R.; Sukumaran, S.K.; Grest, G.S.; Svaneborg, C.; Sivasubramanian, A.; Kremer, K. Rheology and microscopic topology of entangled polymeric liquids. Science 2004, 303, 823-826.

7. Gay, J.; Berne, B. Modification of the overlap potential to mimic a linear site-site potential. J. Chem. Phys. 1981, 74, 3316-3319.

8. Groot, R.D.; Warren, P.B. Dissipative particle dynamics: Bridging the gap between atomistic and mesoscopic simulation. J. Chem. Phys. 1997, 107, 4423.

9. Jury, S.; Bladon, P.; Cates, M.; Krishna, S.; Hagen, M.; Ruddock, N.; Warren, P. Simulation of amphiphilic mesophases using dissipative particle dynamics. Phys. Chem. Chem. Phys. 1999, 1, 2051-2056.

10. Karimi-Varzaneh, H.A.; van der Vegt, N.F.; Müller-Plathe, F.; Carbone, P. How good are coarse-grained polymer models? A comparison for atactic polystyrene. ChemPhysChem 2012, 13, 3428-3439.

11. Kremer, K.; Grest, G.S. Dynamics of entangled linear polymer melts: A molecular-dynamics simulation. J. Chem. Phys. 1990, 92, 5057.

12. Riniker, S.; Allison, J.R.; van Gunsteren, W.F. On developing coarse-grained models for biomolecular simulation: A review. Phys. Chem. Chem. Phys. 2012, 14, 12423-12430.

13. Barducci, A.; Bonomi, M.; Parrinello, M. Metadynamics. Wiley Interdiscip. Rev. 2011, 1, 826-843.

14. Baumgärtner, A.; Binder, K.; Hansen, J.P.; Kalos, M.; Kehr, K.; Landau, D.; Levesque, D.; Müller-Krumbhaar, H.; Rebbi, C.; Saito, Y.; et al. Applications of the Monte Carlo Method in Statistical Physics; Springer Science \& Business Media: Berlin, Germany, 2013; Volume 36.

15. Binder, K. Monte Carlo and Molecular Dynamics Simulations in Polymer Science; Oxford University Press: Oxford, UK, 1995.

16. Rapaport, D.C. The Art of Molecular Dynamics Simulation; Cambridge University Press: Cambridge, UK, 2004.

17. Baig, C.; Mavrantzas, V.G.; Kr'oger, M. Flow effects on melt structure and entanglement network of linear polymers: Results from a nonequilibrium molecular dynamics simulation study of a polyethylene melt in steady shear. Macromolecules 2010, 43, 6886-6902.

18. Barrat, J.L.; Baschnagel, J.; Lyulin, A. Molecular dynamics simulations of glassy polymers. Soft Matter 2010, 6, 3430-3446.

19. Chung, H.S.; Piana-Agostinetti, S.; Shaw, D.E.; Eaton, W.A. Structural origin of slow diffusion in protein folding. Science 2015, 349, 1504-1510.

20. Do, C.; Lunkenheimer, P.; Diddens, D.; Götz, M.; Weiß, M.; Loidl, A.; Sun, X.G.; Allgaier, J.; Ohl, M. $\mathrm{Li}+$ transport in poly (ethylene oxide) based electrolytes: Neutron scattering, dielectric spectroscopy, and molecular dynamics simulations. Phys. Rev. Lett. 2013, 111, 018301.

21. Hossain, D.; Tschopp, M.; Ward, D.; Bouvard, J.; Wang, P.; Horstemeyer, M. Molecular dynamics simulations of deformation mechanisms of amorphous polyethylene. Polymer 2010, 51, 6071-6083. 
22. Hur, K.; Jeong, C.; Winkler, R.G.; Lacevic, N.; Gee, R.H.; Yoon, D.Y. Chain dynamics of ring and linear polyethylene melts from molecular dynamics simulations. Macromolecules 2011, 44, 2311-2315.

23. Mitchell, J.S.; Harris, S.A. Thermodynamics of writhe in DNA minicircles from molecular dynamics simulations. Phys. Rev. Lett. 2013, 110, 148105.

24. Ndoro, T.V.; Voyiatzis, E.; Ghanbari, A.; Theodorou, D.N.; Böhm, M.C.; Müller-Plathe, F. Interface of grafted and ungrafted silica nanoparticles with a polystyrene matrix: Atomistic molecular dynamics simulations. Macromolecules 2011, 44, 2316-2327.

25. Stephanou, P.S.; Baig, C.; Tsolou, G.; Mavrantzas, V.G.; Kröger, M. Quantifying chain reptation in entangled polymer melts: Topological and dynamical mapping of atomistic simulation results onto the tube model. J. Chem. Phys. 2010, 132, 124904.

26. Harmandaris, V.A.; Kremer, K. Dynamics of polystyrene melts through hierarchical multiscale simulations. Macromolecules 2009, 42, 791-802.

27. Pant, P.K.; Han, J.; Smith, G.D.; Boyd, R.H. A molecular dynamics simulation of polyethylene. J. Chem. Phys. 1993, 99, 597-604.

28. Jorgensen, W.L.; Madura, J.D.; Swenson, C.J. Optimized intermolecular potential functions for liquid hydrocarbons. J. Am. Chem. Soc. 1984, 106, 6638-6646.

29. Martin, M.G.; Siepmann, J.I. Transferable potentials for phase equilibria. 1. United-atom description of n-alkanes. J. Phys. Chem. B 1998, 102, 2569-2577.

30. Boyd, R.H.; Gee, R.H.; Han, J.; Jin, Y. Conformational dynamics in bulk polyethylene: A molecular dynamics simulation study. J. Chem. Phys. 1994, 101, 788-797.

31. Harmandaris, V.A.; Mavrantzas, V.G.; Theodorou, D.N. Atomistic molecular dynamics simulation of polydisperse linear polyethylene melts. Macromolecules 1998, 31, 7934-7943.

32. Jin, Y.; Boyd, R.H. Subglass chain dynamics and relaxation in polyethylene: A molecular dynamics simulation study. J. Chem. Phys. 1998, 108, 9912-9923.

33. Kavassalis, T.; Sundararajan, P. A molecular-dynamics study of polyethylene crystallization. Macromolecules 1993, 26, 4144-4150.

34. Moore, J.; Cui, S.; Cochran, H.; Cummings, P. A molecular dynamics study of a short-chain polyethylene melt.: I. steady-state shear. J. Non-Newton. Fluid Mech. 2000, 93, 83-99.

35. Paul, W.; Smith, G.; Yoon, D.Y.; Farago, B.; Rathgeber, S.; Zirkel, A.; Willner, L.; Richter, D. Chain motion in an unentangled polyethylene melt: A critical test of the rouse model by molecular dynamics simulations and neutron spin echo spectroscopy. Phys. Rev. Lett. 1998, 80, 2346.

36. Ramos, J.; Vega, J.F.; Theodorou, D.N.; Martinez-Salazar, J. Entanglement relaxation time in polyethylene: Simulation versus experimental data. Macromolecules 2008, 41, 2959-2962.

37. Rissanou, A.N.; Power, A.J.; Harmandaris, V. Structural and dynamical properties of polyethylene/graphene nanocomposites through molecular dynamics simulations. Polymers 2015, 7, 390-417.

38. Zhang, Y.; Zhuang, X.; Muthu, J.; Mabrouki, T.; Fontaine, M.; Gong, Y.; Rabczuk, T. Load transfer of graphene/carbon nanotube/polyethylene hybrid nanocomposite by molecular dynamics simulation. Compos. Part B Eng. 2014, 63, 27-33.

39. Harmandaris, V.A.; Daoulas, K.C.; Mavrantzas, V.G. Molecular dynamics simulation of a polymer melt/solid interface: Local dynamics and chain mobility in a thin film of polyethylene melt adsorbed on graphite. Macromolecules 2005, 38, 5796-5809.

40. Hu, M.; Keblinski, P.; Schelling, P.K. Kapitza conductance of silicon-amorphous polyethylene interfaces by molecular dynamics simulations. Phys. Rev. B 2009, 79, 104305.

41. Taylor, D.; Strawhecker, K.; Shanholtz, E.; Sorescu, D.; Sausa, R. Investigations of the intermolecular forces between RDX and polyethylene by force-Distance spectroscopy and molecular dynamics simulations. J. Phys. Chem. A 2014, 118, 5083-5097.

42. Hur, K.; Winkler, R.G.; Yoon, D.Y. Comparison of ring and linear polyethylene from molecular dynamics simulations. Macromolecules 2006, 39, 3975-3977.

43. Yi, P.; Locker, C.R.; Rutledge, G.C. Molecular dynamics simulation of homogeneous crystal nucleation in polyethylene. Macromolecules 2013, 46, 4723-4733.

44. Henry, A.; Chen, G. High thermal conductivity of single polyethylene chains using molecular dynamics simulations. Phys. Rev. Lett. 2008, 101, 235502. 
45. Henry, A.; Chen, G. Anomalous heat conduction in polyethylene chains: Theory and molecular dynamics simulations. Phys. Rev. B 2009, 79, 144305.

46. Kim, J.M.; Locker, R.; Rutledge, G.C. Plastic deformation of semicrystalline polyethylene under extension, compression, and shear using molecular dynamics simulation. Macromolecules 2014, 47, 2515-2528.

47. Lavine, M.S.; Waheed, N.; Rutledge, G.C. Molecular dynamics simulation of orientation and crystallization of polyethylene during uniaxial extension. Polymer 2003, 44, 1771-1779.

48. Yeh, I.C.; Andzelm, J.W.; Rutledge, G.C. Mechanical and structural characterization of semicrystalline polyethylene under tensile deformation by molecular dynamics simulations. Macromolecules 2015, 48, 4228-4239.

49. Vu-Bac, N.; Lahmer, T.; Keitel, H.; Zhao, J.; Zhuang, X.; Rabczuk, T. Stochastic predictions of bulk properties of amorphous polyethylene based on molecular dynamics simulations. Mech. Mater. 2014, 68, 70-84.

50. Hess, B.; Bekker, H.; Berendsen, H.J.; Fraaije, J.G. LINCS: A linear constraint solver for molecular simulations. J. Comput. Chem. 1997, 18, 1463-1472.

51. Pronk, S.; Páll, S.; Schulz, R.; Larsson, P.; Bjelkmar, P.; Apostolov, R.; Shirts, M.R.; Smith, J.C.; Kasson, P.M.; van der Spoel, D.; et al. GROMACS 4.5: A high-throughput and highly parallel open source molecular simulation toolkit. Bioinformatics 2013, 29, 845-854.

52. Hoover, W.G. Canonical dynamics: Equilibrium phase-space distributions. Phys. Rev. A 1985, 31, $1695-1697$.

53. Nosé, S. A unified formulation of the constant temperature molecular dynamics methods. J. Chem. Phys. 1984, 81, 511-519.

54. Hockney, R.W. The potential calculation and some applications. Methods Comput. Phys. 1970, 9, $135-211$.

55. Flory, P.J. The configuration of real polymer chains. J. Chem. Phys. 1949, 17, 303-310.

56. Hoy, R.S.; Foteinopoulou, K.; Kröger, M. Topological analysis of polymeric melts: Chain-length effects and fast-converging estimators for entanglement length. Phys. Rev. E 2009, 80, 031803.

57. Kröger, M. Shortest multiple disconnected path for the analysis of entanglements in two-and three-dimensional polymeric systems. Comput. Phys. Commun. 2005, 168, 209-232.

58. Shanbhag, S.; Kröger, M. Primitive path networks generated by annealing and geometrical methods: Insights into differences. Macromolecules 2007, 40, 2897-2903.

59. Rouse, P.E., Jr. A theory of the linear viscoelastic properties of dilute solutions of coiling polymers. J. Chem. Phys. 1953, 21, 1272-1280.

60. Likhtman, A.E.; Sukumaran, S.K.; Ramirez, J. Linear viscoelasticity from molecular dynamics simulation of entangled polymers. Macromolecules 2007, 40, 6748-6757.

(C) 2017 by the authors; licensee MDPI, Basel, Switzerland. This article is an open access article distributed under the terms and conditions of the Creative Commons Attribution (CC-BY) license (http://creativecommons.org/licenses/by/4.0/). 